\title{
US concern grows over secrecy clauses
}

[воSTON] A US physician told a conference on secrecy in science this week that he is to lose his job because of claims that he broke a 'non-disclosure' agreement over findings about a potentially fatal occupational lung disease among a group of textile workers.

David Kern, from Brown University, Rhode Island, was speaking at a meeting on Monday (29 March) at Massachusetts Institute of Technology (MIT). Representatives from universities, industry and government had met to address an issue of growing concern as increasing numbers of university researchers form links with private industry.

Two years ago, Kern was told by a Rhode Island textile manufacturer for whom he had done consultancy work that he would be sued if he and his colleagues presented their findings about a lung disease they had identified among company workers. The company claimed that Kern was prohibited from publicizing his research owing to a non-disclosure agreement he had signed.

Kern maintains that the agreement only pertained to a one-hour visit to the plant that he and medical students had made 15 months before the research began. Kern's research contract with the company stated that "we will report to the scientific and public health communities as we deem fit".

But, according to Kern, Brown University and the Memorial Hospital of Rhode Island at which he works, sided with the company, and his contract with both the hospital and university will be terminated in three months.

The conference, sponsored by MIT and the American Association for the Advancement of Science, examined how university-industry agreements can affect the conduct and scope of scientific research, and the dissemination of findings.

"Secrecy is not always bad," said John Deutch, MIT professor and former director of the Central Intelligence Agency. "Indeed, the privacy of individuals should be protected. But secrecy is a major threat to science and is antithetical to the purposes of universities."

Of particular concern to conference participants were university-industry relationships that could lead to the suppression of research results, delay the publication of data, or otherwise restrict the sharing of information and research tools to allow a company or individuals to preserve a competitive edge.

"Many people are increasingly fearful that the 'non-disclosure agreements' imposed on scientists by industry are fundamentally distorting the way science is done," said Boyce Rensberger, formerly a reporter at The New York Times and Washington Post, and now director of the Knight Science Journalism Fellowship programme at MIT.

Rensberger believes that non-disclosure

NATURE| VOL 398 | 1 APRIL 1999 |www.nature.com agreements have had a chilling effect on science. "I used to argue that we could count on academic scientists to tell us the truth because they were independent and honest, but nowadays I'm not so sure," he said.

"A fair number of them are subject to gag rules, often saying, 'Sorry I can't tell you; that's proprietary'. When you interview a scientist, you now have to ask: 'Have you signed a non-disclosure agreement that prevents you from speaking freely?' Then you have to decide whether you can trust the answer."

Whether because of non-disclosure agreements or the lure of financial gain, significant numbers of scientists are withholding data or delaying its publication. Alan Goldhammer of the Biotechnology Industry Organization said the problem cannot be blamed entirely on greedy corporations. "Part of the fault lies with the academics who

think, 'By God, I'm going to be rich.' But Howard Schachman, of the University of California, Berkeley, argued that money has had an unquestionably disruptive influence.

"Why do you need a licence to obtain a reagent from a colleague at another university?" he asked. "The increasing commercialization of research has blurred the line between research tools [such as the polymerase chain reaction] and products."

Although no firm solutions emerged from the conference, the general consensus was that the fruits of research should be shared as quickly as possible to benefit both science and society.

Lita Nelsen, director of MIT's Technology Licensing Office, said academic institutions should establish policies to protect faculty members who lack the power to set appropriate terms with companies.

Steve Nadis

\section{UK research councils look to future needs}

[LONDON] The heads of Britain's main research councils agreed on Tuesday (30 March) to 'steer' a group developing a long-term technology strategy for the whole science base as part of the government's Foresight initiative.

The overall aim of the initiative, launched in 1993, is to stimulate the thinking of scientists and industrialists about how science could help to deliver the products and services of the future, focusing on technologies that create wealth and improve the quality of life.

So far, some areas of science - such as particle physics - have played virtually no role in the Foresight process. Following consultation with the scientific community, however, the government's Office of Science and Technology (OST) recently created 'associate programmes' to allow special interest groups, such as learned societies and research councils, to play a wider role.

A working group of research council officials has already formed a partnership to develop proposals for an associate programme known as the Long-term Technology Review. This will examine the technologies required in five or more years' time to maintain a strong research base in the United Kingdom.

Over the next six months, each research council will analyse its future requirements, based on its science objectives, and produce a statement on its technology needs. These will be drawn into a common report identifying the needs of the science base in specific technical areas, such as detector systems, electronics, materials, biochips and information technology.

This report will allow other Foresight

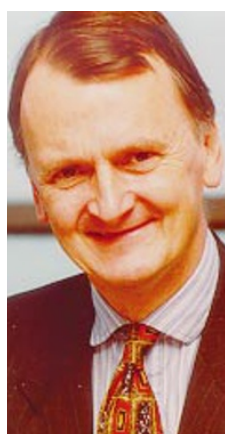

Halliday: exciting collaboration. panels to take into account the needs of the science base, and enable common areas of interest to be identified across the science community. An open consultation will also enable researchers to contribute ideas on future technology requirements.

The Particle Physics and Astronomy Research Council (PPARC) has been invited by the OST to lead the associate programme on the technology needs of the science base on the strength of its work last year on a long-term technology plan for particle physics and astronomy.

Peter Fletcher, PPARC's head of industrial and international liaison, says the first Foresight exercise "did not give us the opportunity to offer solutions to other people's technology problems, although our research requirements are so demanding we are at the cutting edge of technology".

Ian Halliday, PPARC's chief executive, says that the current exercise is "an opportunity for the academic and university community to get into print their requirements. This is a serious mechanism for collaboration between different research councils. We are very excited about it."

The technology review will run for two years and Fletcher hopes that by the end of the process it will have been possible to "create a new understanding of common technology interests between the science base and industry". 